\title{
Functional analysis of the impact of ORMDL3 expression on inflammation and activation of the unfolded protein response in human airway epithelial cells
}

Karolynn J Hsu and Stuart E Turvey*

\begin{abstract}
Background: The gene ORMDL3 was shown to be associated with early-onset asthma susceptibility in multiple independent genome-wide and candidate-gene association studies. Asthmatic patients have elevated expression levels of this gene. ORMDL3 encodes a transmembrane protein localized in the endoplasmic reticulum (ER) that may be involved in ER stress and inflammation. It is essential to validate the genetic associations linking ORMDL3 with asthma through functional studies that confirm the biological relevance of this gene in disease. We investigated the effects of manipulating ORMDL3 expression levels in vitro in airway cells on innate immune inflammatory responses, ER stress and activation of the unfolded protein response (UPR).
\end{abstract}

Methods: ORMDL3 expression levels were manipulated in airway cells using an overexpression plasmid and siRNA technologies. Successful modulation of ORMDL3 was confirmed at both the gene and protein level. The functional impact of modulation of ORMDL3 expression levels on inflammatory responses and activation of the UPR were quantified using complementary cellular and molecular immunology techniques.

Results: Cells with altered ORMDL3 levels responded equally well to innate immune stimuli and produced similar levels of pro-inflammatory cytokines compared to wild-type cells. Treatment with ER stress inducers, thapsigargin and tunicamycin, resulted in activation of the unfolded protein response (UPR). However, we observed no difference in UPR activation in cells with ORMDL3 knockdown compared to cells with normal ORMDL3 levels.

Conclusions: Our results suggest that ORMDL3 variation in the airway epithelium is unlikely to play a significant role in modulating innate immune responses and the UPR in the lung.

Keywords: Immune response, Epithelium, Cytokines, Chemokines, Host defense

\section{Introduction}

Asthma and allergic diseases are rapidly becoming the most common chronic diseases in the developed world. Current asthma therapy treats symptoms of the disease, however it is ineffective in up to $25 \%$ of patients [1]. Asthma and allergic diseases are complex disorders caused by the interaction of various genetic and environmental factors [2-4].

\footnotetext{
* Correspondence: sturvey@cfri.ca

Division of Infectious and Immunological Diseases, Department of Pediatrics, BC Children's Hospital and Child \& Family Research Institute, University of
} British Columbia, Vancouver, BC, Canada
Genome-wide association studies (GWAS) have been used to identify genes that may be involved in asthma pathogenesis [5]. Moffatt and colleagues first reported that multiple single nucleotide polymorphisms (SNPs) on chromosome 17q21 linked ORMDL3 (orosomucoid 1-like 3) to the risk of developing childhood asthma [6]. This association has since been reproduced in multiple independent studies [7-14]. However, little work has been done to elucidate the biological and functional relevance of this gene in asthma. The disadvantage of these association studies is that they cannot differentiate between true causal SNPs and non-causal variants simply in linkage disequilibrium with disease-causing genes. It 
is therefore imperative to validate GWAS data through functional studies that confirm the biological relevance of a gene in disease.

SNP variants have also linked ORMDL3 to inflammatory bowel disease (IBD) and Type I diabetes, suggesting that ORMDL3 may be involved in dysregulation of the immune system $[15,16]$. Association of ORMDL3 in both asthma and IBD is of interest because the lung and gut are composed of similar mucosal surface cells and these tissues are exposed to many potentially harmful antigens and allergens requiring tight regulation of the mucosal immune system [17]. This unique system is responsible for maintaining a delicate equilibrium between antigen responsiveness and tolerance and is therefore responsible for preventing hyper-reactivity [17]. Inappropriate immune responses to foreign components or commensal bacteria can lead to inflammation characteristic of asthma and IBD. Furthermore, the polymorphisms may be involved in regulation of mRNA expression of 17q21 locus genes, including ORMDL3 [6]. The expression of ORMDL3 was recently associated with elevated levels of IL-17 secretion [18] and $O R M D L 3$ was expressed at higher levels in the peripheral blood of patients with recurrent wheeze compared to controls [19]. This correlation further supports the hypothesis that $O R M D L 3$ is involved in immunity.

The ORMDL3 gene is a member of a family of conserved endoplasmic reticulum (ER)-localized transmembrane proteins [20]. The functions of the ORMDL proteins are currently unknown, but a recent study suggested that ORMDL3 is involved in ER-mediated $\mathrm{Ca}^{2+}$ homeostasis and activation of the unfolded protein response (UPR) ORMDL3 may inhibit sarco/endoplasmic reticulum $\mathrm{Ca}^{2+}$ ATPase (SERCA) activity [21,22]. Disruptions to ER $\mathrm{Ca}^{2+}$ concentrations can cause protein misfolding, and accumulation of these unfolded proteins can lead to ER stress $[23,24]$. UPR signaling cascades are initiated in response to this stress and have been shown to activate the JNK-AP-1 and NF-kB-IKK pathways [25-27]. The ER stress response and UPR, caused by changes in ORMDL3 expression, can initiate inflammation through induction of cytokine production. This mechanism may explain the role of ORMDL3 in asthma pathogenesis. Indeed, Miller et al. have shown that in mice ORMDL3 is an allergen and cytokine (IL-4 or IL-13) inducible ER gene expressed predominantly in airway epithelial cells, and that it activates the ATF6 pathway of the ER localized UPR regulating expression of metalloprotease, chemokine, and oligoadenylate synthetase genes [28].

Although the symptoms of asthma are largely driven by dysregulated $\mathrm{T}$ helper type $2\left(\mathrm{~T}_{\mathrm{H}} 2\right)$ responses, innate immune responses are also involved in asthma pathogenesis $[29,30]$. Airway epithelia are central to host defense and immune regulation. These cells are among the first to encounter environmental insults and play an important role in shaping downstream immune responses. Any dysregulation of the innate immune response can result in hypersensitivity to environmental factors, leading to asthma symptoms.

Given the multiple lines of evidence suggesting that $O R M D L 3$ is involved in immunity, we investigated the role of the gene in innate immune responses of airway cells. We hypothesized that elevated ORMDL3 levels result in heightened inflammatory responses that are associated with the asthmatic phenotype. Increased levels of ORMDL3 protein may in turn disrupt ER homeostasis, leading to ER overload and activation of the UPR, initiating inflammatory responses. Using an in vitro model, we manipulated $O R M D L 3$ expression in airway cells to determine whether a difference in basal ORMDL3 expression affected inflammatory responses or activation of the UPR before and after stimulation.

\section{Materials and methods Cell culture}

1HAEo (1HAE) cells (SV40-transformed normal human airway epithelial cells) were cultured in DMEM-high glucose medium with $10 \%$ fetal calf serum (FCS), $2 \mathrm{mM} \mathrm{L-}$ glutamine, and $1 \mathrm{mM}$ sodium pyruvate (HyClone). A549 cells (adenocarcinomic human alveolar basal epithelial cells) were cultured in F-12K medium supplemented with $10 \%$ fetal calf serum (FCS), $2 \mathrm{mM}$ L-glutamine, and $1 \mathrm{mM}$ sodium pyruvate (HyClone). Cells were incubated in a $37^{\circ} \mathrm{C}$, $5 \% \mathrm{CO}_{2}$ incubator. All cells were cultured under nonpolarizing conditions.

\section{Cloning ORMDL3 CDNA into pEGFP-N1 vector}

The ORMDL3 gene was amplified from cDNA using forward primer 5'-CTAAGAATTCATGAATGTGGGCAC AGCGCAC- $3^{\prime}$ and reverse primer $5^{\prime}$-TACTGGTACCCC GTACTTATTGATTCCAAAAATCCGGACT-3', introducing EcoRI and $K p n I$ restriction endonuclease sites, respectively. The ORMDL3 PCR product was then inserted into a pEGFP-N1 eukaryotic expression vector (Clontech). $O R M D L 3$ and $e G F P$ are in frame and produce a fusion protein with eGFP expressed at the C-terminus of ORMDL3. The construct was verified by sequencing and is denoted as pEGFP-ORMDL3. Protein is denoted as ORMDL3-eGFP.

\section{Cell transfection}

A549 and 1HAE cell lines were transfected with pEGFPORMDL3, scramble (non-specific) or ORMDL3-specific siRNA (pre-designed by Qiagen) using Amaxa $^{\circledR}$ Cell Line Nucleofector ${ }^{\circledR}$ Kit T (Lonza). Two ORMDL3-specific siRNAs were used. Concentrations used for transfection represent pooled siRNA concentration. Cells were seeded into a 24-well plate (BD Biosciences) at a 
density of $2 \times 10^{5}$ cells/well for A549 cells or $1 \times 10^{5}$ cells/ well for $1 \mathrm{HAE}$ cells.

\section{Cell stimulation and immune response quantification}

Twenty-four hours post-transfection, cells were stimulated with TNF- $\alpha(200 \mathrm{ng} / \mathrm{ml})$ (eBioscience), E. coli K12 LPS $(100 \mu \mathrm{g} / \mathrm{ml})$ (InvivoGen), S. typhimurium flagellin $(10-200 \mathrm{ng} / \mathrm{ml})$ (InvivoGen), or IL-1 $\beta(200 \mathrm{ng} / \mathrm{ml})$ (eBioscience). Stimulants and their concentrations were chosen based on published literature or past experiments [31-34]. Cells were stimulated for 24 hours. Supernatants were collected and analyzed for cytokine secretion. Proinflammatory cytokines, IL- 6 and IL-8, were detected and quantified using Human IL-6 and IL-8 Ready-Set-Go! ${ }^{\circledR}$ ELISA kits (eBioscience). Experiments were repeated three times $(\mathrm{n}=3)$.

\section{ER stress induction and UPR activation}

Cells were stimulated with tunicamycin $(200 \mu \mathrm{g} / \mathrm{mL})(\mathrm{Cal}-$ biochem) or thapsigargin $(10 \mu \mathrm{M})$ (Sigma) for 2 or 4 hours to activate the UPR. For ORMDL3 knockdown cells, stimulation was performed 24 hours post-transfection. RNA was extracted and expression of genes $X B P-1 u$, $X B P-1 s$, and $C H O P$ were then quantified as markers of UPR activation. For measurement of p-eIF2 $\alpha$ levels by Western blot, lysates from unstimulated cells with ORMDL3 knockdown were collected 24 hours post-transfection. Experiments were repeated three times $(\mathrm{n}=3)$.

\section{RNA isolation and reverse transcription}

RNA was extracted from lysates using E.Z.N.A. ${ }^{\circledR}$ Total RNA Kit (Omega Bio-Tek) according to the manufacturer's protocol. Extracted RNA was reverse transcribed into cDNA using the SuperScript ${ }^{\circledR}$ VILO $^{\mathrm{TM}}$ cDNA Synthesis Kit (Invitrogen). Complement DNA was diluted to $200 \mathrm{ng} / \mu \mathrm{l}$ prior to quantification of gene expression by qPCR. This method was followed for all samples, unless otherwise stated (see PCR Array).

\section{Quantification of ORMDL3 mRNA expression}

Gene expression was calculated relative to GAPDH or PPIA (encoding cyclophilin A) and was quantified by SYBR Green chemistry (PerfeCTa ${ }^{\mathrm{TM}}$ qPCR SuperMix, Quanta Biosciences) using a 7300 Real Time PCR System (Applied Biosystems). Reactions were performed in triplicate using the following cycling conditions: $50^{\circ} \mathrm{C}$ for 2 mins, $95^{\circ} \mathrm{C}$ for 10 mins, [ $95^{\circ} \mathrm{C}$ for $15 \mathrm{~s}, 60^{\circ} \mathrm{C}$ for $1 \mathrm{~min}$ ] $\mathrm{x} 40$. The relative expression of the measured gene was calculated by the Pfaffl method [35]. The primers used are listed in Table 1.

\section{Western blot analysis}

Cells were lysed in $50 \mu \mathrm{l}$ RIPA Buffer $+1 \mathrm{x}$ HALT $^{\mathrm{TM}}$ protease inhibitor (Thermo Scientific). Cell debris were removed by centrifugation: $18,000 \times \mathrm{g}$ for $10 \mathrm{~min}$ at $4^{\circ} \mathrm{C}$. Proteins were analyzed by standard Western blotting protocols where they were transferred onto Immobilon ${ }^{\circledR}$-FL transfer membrane (Millipore). Antibodies used for Western blot

Table 1 Quantitative PCR primer sequences

\begin{tabular}{|c|c|c|c|c|c|}
\hline Gene & NCBI accession & Forward $\left(5^{\prime} \rightarrow 3^{\prime}\right)$ & Reverse $\left(5^{\prime} \rightarrow 3^{\prime}\right)$ & CDS region $^{\dagger}$ & Product size \\
\hline GAPDH & NM_002046.4 & GCACCGTCAAGGCTGAGAACGG & CGACGTACTCAGCGCCAGCATC & c. $173-286$ & 114 \\
\hline PPIA & NM_021130 & TAAAGCATACGGGTCCTGGCATCT & ATCCAACCACTCAGTCTTGGCAGT & c. $269-369$ & 101 \\
\hline$A C T B$ & NM_001101.3 & GTTGCGTTACACCCTTTCTT & ACCTTCACCGTTCCAGTTT & c. ${ }^{*} 16-{ }^{*} 162$ & 147 \\
\hline DDIT3 & NM_001195053.1 & GAAATGAAGAGGAAGAATCA & TTCTCCTTCATGCGCT & c. $197-437$ & 241 \\
\hline$X B P-15$ & NM_001079539.1 & ATGGATGCCCTGGTTGCTGAAGA & TGCACCTGCTGCGGACTCA & c. $415-504$ & 90 \\
\hline$X B P-1 U$ & NM_005080.3 & AGCACTCAGACTACGTGCACCTCT & CCAGAATGCCCAACAGGATATCAG & c. $495-624$ & 130 \\
\hline ORMDL1 & NM_016467.4 & AATGGCTGGTCCTTCAAGTGCT & ACCCTCACTGTGATGCCCTITA & c.*121-*269 & 149 \\
\hline ORMDL2 & NM_014182.4 & ACACACTGGGAGCAAATGGACT & AGTGCGCAGCATCATACTTGGT & c. $250-370$ & 121 \\
\hline ORMDL3 & NM_139280.2 & TCAGGCAGCCAAAGCACTITAACC & ACCCATCCCACACTTGCTTCCATA & c. ${ }^{*} 358-* 496$ & 139 \\
\hline$B C L 6$ & NM_001706.4 & ACAATCCCAGAAGAGGCACGAAGT & GCTCGAAATGCAGGGCAATCTCAT & c.790-952 & 163 \\
\hline CCL2 & NM_002982.3 & TCGCTCAGCCAGATGCAATCAATG & TGGAATCCTGAACCCACTTCTGCT & c. $65-259$ & 195 \\
\hline CCL5 & NM_002985.2 & TGCCTGTTCTGCTTGCTCTTGTC & TGTGGTAGAATCTGGGCCCTTCAA & c. ${ }^{*} 36-{ }^{*} 127$ & 92 \\
\hline CSF2 & NM_000758.3 & AAATGTITGACCTCCAGGAGCCGA & GGTGATAATCTGGGTTGCACAGGA & c. $185-357$ & 173 \\
\hline IL12A & NM_000882.3 & ATGATGGCCCTGTGCCTTAGTAGT & AGGGCCTGCATCAGCTCATCAATA & c.457-611 & 155 \\
\hline IL13RA1 & NM_001560.2 & GTCCCAGTGTAGCACCAATGA & CAGTCACAGCAGACTCAGGAT & c.297-391 & 95 \\
\hline ADRB2 & NM_000024.5 & TCATCATGGGCACTTTCACCCTCT & AGCTCCTGGAAGGCAATCCTGAAA & c.830-1016 & 187 \\
\hline VEGFA & NM_001025366.2 & TTCAGGACATTGCTGTGCTTTGGG & TGGGCTGCTTCTTCCAACAATGTG & c.*778-*969 & 192 \\
\hline IL23A & NM_016584.2 & TCGGTGAACAACTGAGGGAACCAA & TGGAATCTCTGCCCACTTCCACTT & C. $-140--54$ & 87 \\
\hline
\end{tabular}

${ }^{\dagger}$ c.-number if in the $5^{\prime} U T R, c .{ }^{*}$ number if in the $3^{\prime} U T R$. 
analysis were: monoclonal anti-GFP antibody 1:10,000 (Clontech), anti-ACTB antibody 1:6,000 (Cell Signaling), anti-p-eIF2 $\alpha$ 1:500 (Cell Signaling) and IRDye ${ }^{\circledR} 680$ or 800 secondary antibodies 1:8000 (Li-cor). Western blots were visualized using an Odyssey Infrared Imaging System (Li-cor).

\section{PCR array}

1HAE cells co-transfected with pEGFP-ORMDL3 and ORMDL3 siRNA (low ORMDL3 expression) were compared to cells co-transfected with pEGFP-ORMDL3 and scramble siRNA (high ORMDL3 expression) at two timepoints ( 2 and 24 hours) after TNF- $\alpha$ stimulation. Extracted RNA was reverse transcribed into first strand CDNA using the RT ${ }^{2}$ First Strand Kit (SABiosciences, Qiagen). Protocol as described by the manufacturer was followed.

Two $\mathrm{RT}^{2}$ Profiler PCR arrays (SABiosciences, Qiagen), profiling expression of 84 genes each, were used: Human Cytokines \& Chemokines and Allergy \& Asthma (see Additional file 1 for complete list). Complementary DNA template was mixed with $\mathrm{RT}^{2} \mathrm{SYBR}^{\circledR}$ Green qPCR Mastermix (SABiosciences, Qiagen) as follows: $1350 \mu \mathrm{L}$ SYBR Green Master Mix, $1248 \mu \mathrm{L}$ nuclease-free $\mathrm{H}_{2} \mathrm{O}$, and $102 \mu \mathrm{L}$ cDNA ( 200 ng/ $\mu \mathrm{L})$. Note: these volumes were used as recommended by the manufacturer for use with a 7300 Real Time PCR System (Applied Biosystems). Template was then aliquoted into PCR plates containing predispensed primers. Cycler program as provided by the manufacturer was used. Results were analyzed using the PCR Array Data Analysis Web Portal.

\section{Statistical analysis}

Data are shown as mean \pm SEM of three separate experiments. Results were analyzed using one-way ANOVA with Bonferroni post-test. Statistical analysis was performed using GraphPad Prism5 (GraphPad Software, Inc.). Differences with $\mathrm{p}<0.05$ were considered significant.

\section{Results}

\section{ORMDL3 modulation in airway cells}

In order to determine functional impact of ORMDL3 modulation, knockdown of the gene was performed using siRNAs. A549 and 1HAE cells were transfected with scramble (non-specific) or ORMDL3-specific siRNA. Modeling variation expected to occur in the human population, we achieved 40-70\% knockdown of ORMDL3 gene expression using siRNA concentrations of $50 \mathrm{nM}-500 \mathrm{nM}$. We also confirmed that $O R M D L 3$-specific siRNA did not affect expression of genes in the same family, ORMDL1 or ORMDL2 (Additional file 2). Sequences of primers used for qPCR are listed in Table 1.

ORMDL3 has $84 \%$ and $80 \%$ protein sequence homology to ORMDL1 and ORMDL2, respectively. This presented a challenge for confirming knockdown of ORMDL3 protein because commercially available antibodies detect all three proteins. Therefore, we were unable to show ORMDL3 protein knockdown in cells transfected with siRNA alone. To overcome this limitation, we constructed a fusion protein, where ORMDL3 is tagged with an eGFP protein. Co-transfection of this overexpression plasmid with ORMDL3-specific siRNA enabled us to knockdown ORMDL3 and detect changes at the protein level using an anti-GFP antibody.

Airway cells were co-transfected with both pEGFPORMDL3 and siRNA (scramble or ORMDL3). Protein and mRNA were analyzed for gene knockdown. At the transcript level, we observed (Figure 1) a small but significant increase $(\mathrm{p}<0.05)$ in ORMDL3 expression in the cells transfected with pEGFP-ORMDL3 and scramble siRNA compared to cells alone, confirming successful overexpression of the ORMDL3 gene. Transfection with pEGFPORMDL3 and increasing concentrations of ORMDL3 siRNA resulted in a titration effect of increasing ORMDL3 knockdown. One advantage to constructing a fusion protein, ORMDL3-eGFP, is that eGFP is only expressed with expression of ORMDL3. Therefore, when cells are cotransfected with pEGFP-ORMDL3 and siRNA, knockdown of ORMDL3 protein could subsequently be detected by immunoblot analysis using the anti-GFP antibody. Expression of the ORMDL3-eGFP protein was confirmed by

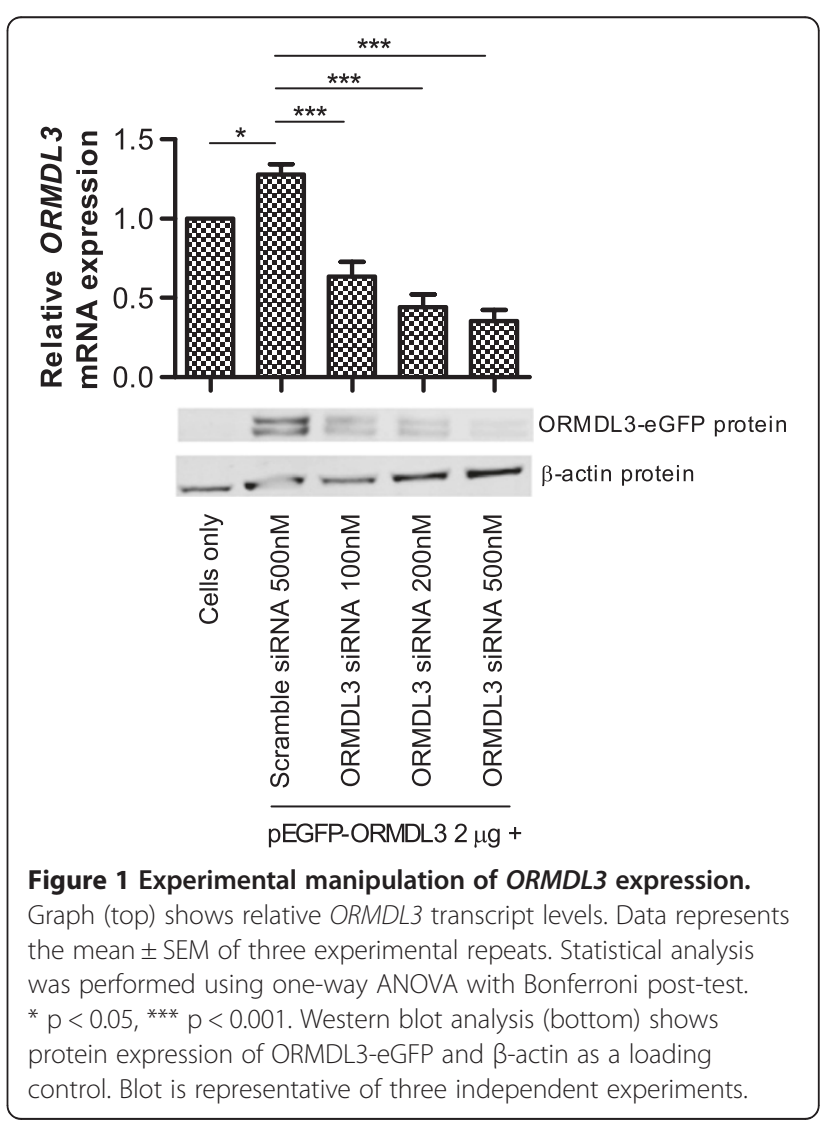


Western blot of whole cell lysate collected from the cells in each condition. Figure 1 shows knockdown of ORMDL3-eGFP protein, confirming that ORMDL3 siRNA affects protein expression. The double band may be explained by either variation in mRNA splicing or posttranslational modifications to the fusion protein, such as acetylation, methylation, myristylation, phosphorylation, or glycosylation.

\section{ORMDL3 knockdown does not affect IL-6 or IL-8 production following innate immune activation}

$1 \mathrm{HAE}$ cells transfected with pEGFP-ORMDL3 and scramble or ORMDL3-specific siRNA were stimulated 24 hours post-transfection and supernatants were collected 48 hours post-transfection. Stimuli used were TNF- $\alpha$, IL-1 $\beta$, LPS, and flagellin. TNF- $\alpha$ and IL-1 $\beta$ were chosen because both are early response cytokines that perpetuate acute inflammatory processes. LPS and flagellin, in contrast, are common microbial antigens recognized by the innate immune system. Two classic and biologically-relevant NF-kB-induced cytokines with important roles in innate immunity, interleukin-6 (IL-6) and interleukin-8 (IL-8), were measured by ELISA. Despite confirmation of ORMDL3 mRNA and protein knockdown, we did not observe any impact on IL-6 or IL-8 production after stimulation as shown in Figure 2A-B. Although the cells have low baseline responsiveness to LPS and flagellin, our results indicate that ORMDL3 knockdown does not enhance sensitivity to these stimuli. Similar results were obtained in A549 cells, as well as 1HAE cells transfected with siRNA alone (Additional file 2).

\section{ORMDL3 knockdown does not enhance UPR activation upon stimulation}

We next explored the effects of ORMDL3 expression on activation of the UPR. Initiation of the UPR is mediated by one or more of the ER-membrane protein sensors: PKR-like eukaryotic initiation factor $2 \alpha$ kinase (PERK), inositol requiring enzyme 1 (IRE1), and activating transcription factor-6 (ATF6) [23]. Activation of any of the three pathways initiates signaling cascades that mediate changes to relieve ER stress. The gene $X B P-1$ is a substrate for IRE1 ribonuclease [24]. Upon activation of the IRE1 pathway, the IRE1 ribonuclease removes a 26-bp intron from the unspliced variant, $X B P-1 u$, which results in the spliced variant, $X B P-1 s$ [36]. This spliced variant is the active form of the gene that contributes to ER stress responses. CHOP transcription, in contrast, can be induced by the PERK and ATF6 pathways [24]. Phosphorylated eIF $2 \alpha$ (p-eIF $2 \alpha$ ) is an early marker of PERK pathway activation and is upstream of $C H O P$ induction [23]. Expression changes in these three genes that signify UPR activation, $X B P-1 u, X B P-1 s$, and $C H O P$, were determined by qPCR. We also evaluated phosphorylation of eukaryotic initiation factor $2 \alpha(\mathrm{eIF} 2 \alpha)$ by Western blot, as modulation of ORMDL3 expression has been reported to influence eIF2 $\alpha$ phosphorylation [21].
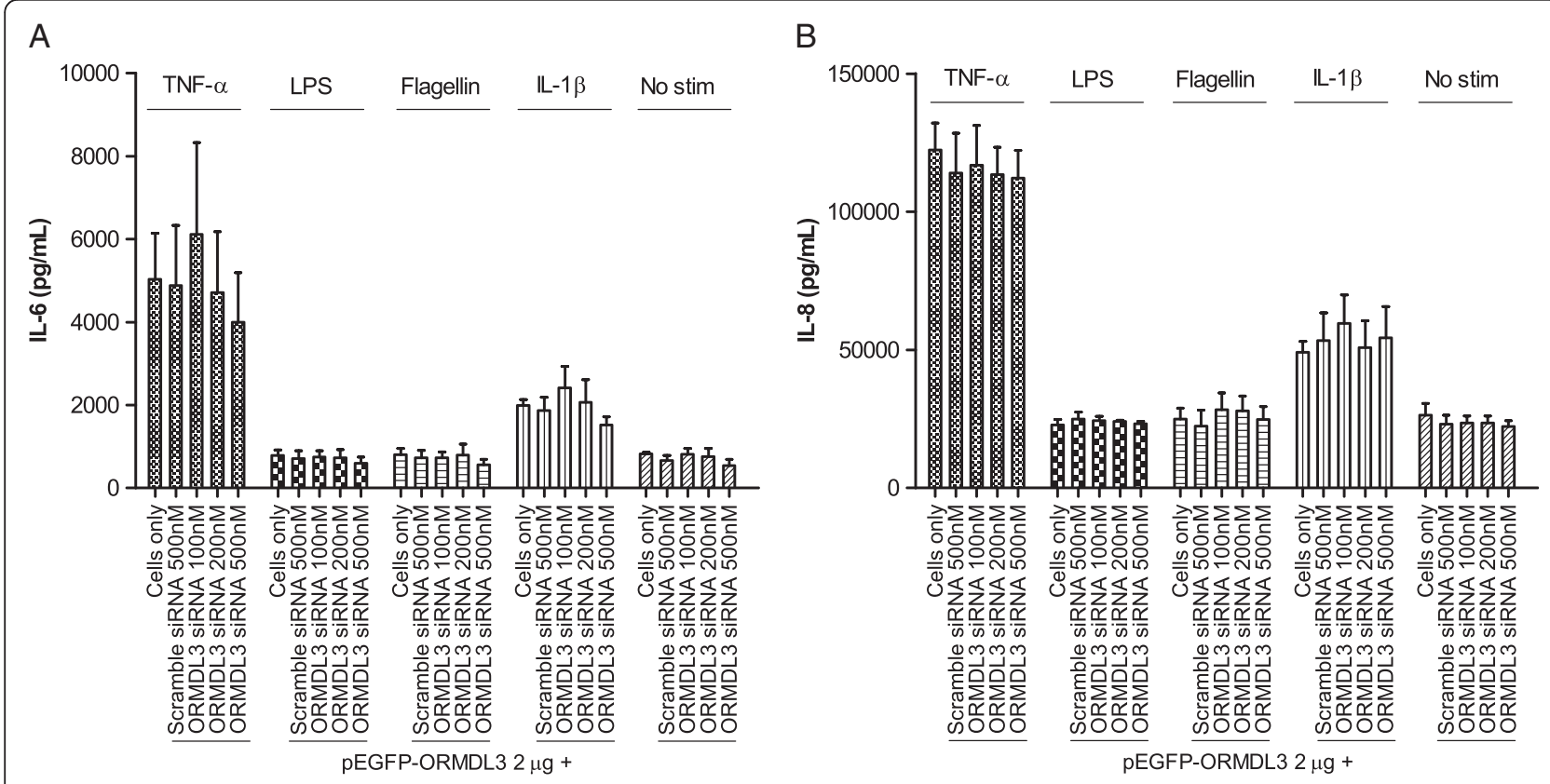

Figure 2 Cytokine production in cells with ORMDL3 knockdown. A) Secreted IL-6 and B) IL-8 levels after cell stimulation. 1HAE cells were stimulated with TNF-a, LPS, flagellin, or IL-1 $\beta$ for 24 hours. Data represent the mean \pm SEM of three experimental repeats. Statistical analysis was performed using one-way ANOVA with Bonferroni post-test. 
As positive controls for UPR activation, we stimulated 1HAE cells with tunicamycin and thapsigargin. Both are inducers of ER stress - tunicamycin inhibits N-linked glycosylation and thapsigargin inhibits the SERCA pump causing ER calcium stores to be depleted [37]. Quantitative measurement of transcript levels showed that both $X B P-1 s$ and $C H O P$ increased, while $X B P-1 u$ decreased upon stimulation with either tunicamycin or thapsigargin (Figure 3A-B). This confirmed the utility of measuring these genes to monitor UPR activation.
At baseline (unstimulated cells), variation in ORMDL3 expression did not induce UPR activation in A549 or $1 \mathrm{HAE}$ cells (Additional file 2). 1HAE cells with ORMDL3 knockdown were also stimulated with tunicamycin or thapsigargin (Figure $3 \mathrm{C}-\mathrm{D}$ ). In both conditions, ORMDL3 knockdown did not show increased UPR activation compared to the negative control. Furthermore, levels of phosphorylated-eIF $2 \alpha$ were indistinguishable between ORMDL3 knockdown cells and controls (Figure 3E).

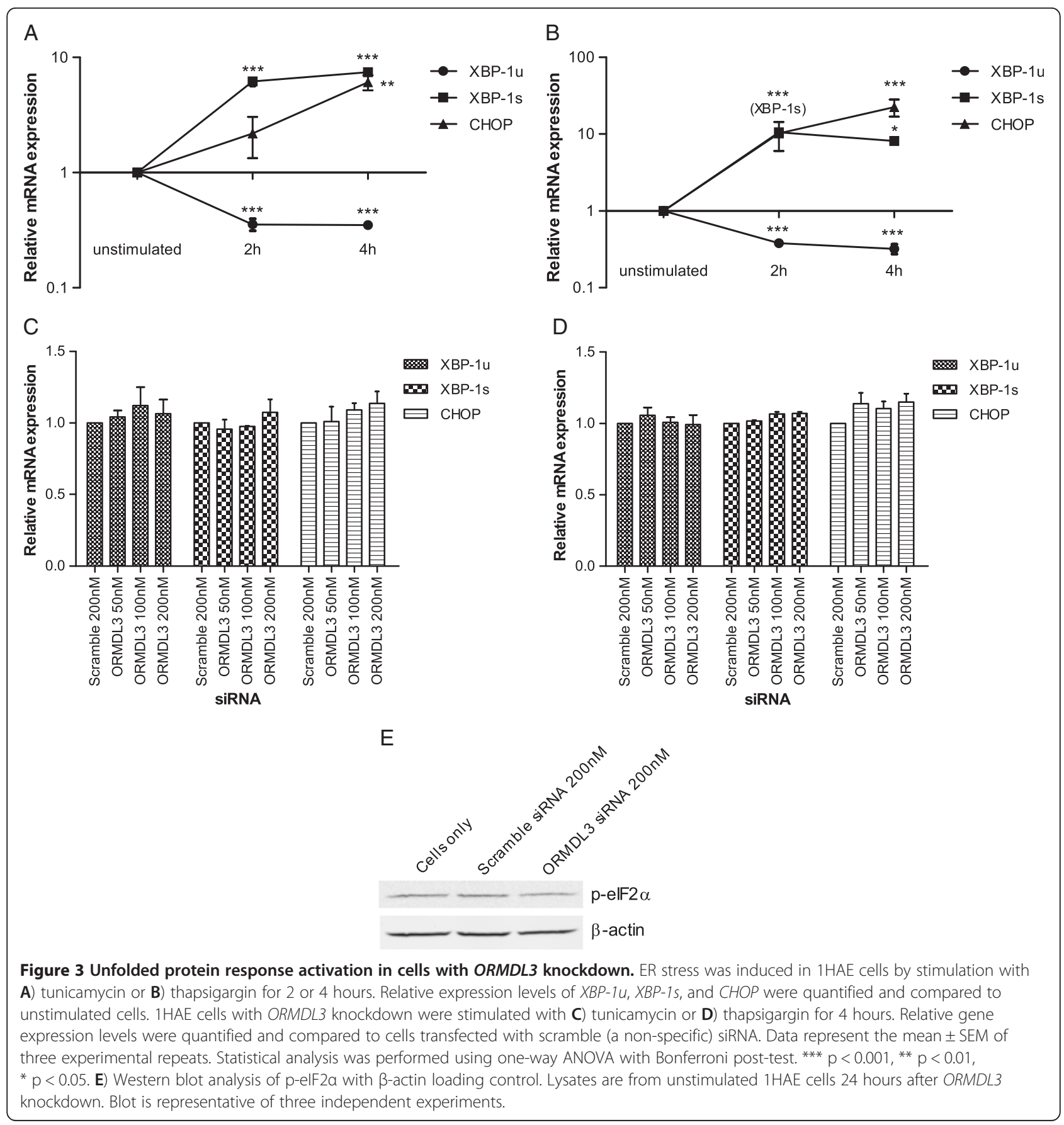


Impact of ORMDL3 knockdown on the expression of multiple genes involved in inflammation, asthma \& allergy

To expand our search for immune functions potentially altered by ORMDL3, the expression of 168 genes was determined at two time points ( 2 and 24 hours) following stimulation with TNF- $\alpha$. We performed PCR arrays to profile expression of cytokines, chemokines, and key genes involved in asthma and allergy (a complete list of genes that were studied can be found in Additional file 1).

Gene expression was compared between $1 \mathrm{HAE}$ cells with high (plasmid + $500 \mathrm{nM}$ scramble siRNA) and low (plasmid + $500 \mathrm{nM}$ of ORMDL3 siRNA) ORMDL3 expression that were stimulated for 24 hours with TNF- $\alpha$. Stimulation with TNF- $\alpha$ induced a robust inflammatory response in the cells, enabling us to observe whether variation in basal ORMDL3 levels impacts the immune response. These arrays identified eight genes (CCL2, TSLP, CSF2, CCL5, VEGFA, ADRB2, IL1RL1, and IL13RA1), shown in Figure 4, that appeared to be differentially regulated by more than 1.5 fold and that were expressed at relatively high levels (average threshold cycle <30). However, upon replication to validate these results, we determined that the differences were not statistically significant.

The same arrays were performed on cells stimulated for 2 hours with TNF- $\alpha$. The same conditions for "high" and "low" ORMDL3 expression were used. From our results, we identified only one gene, IL23A, that was differentially regulated by more than 1.5 fold (fold regulation -2.37 )

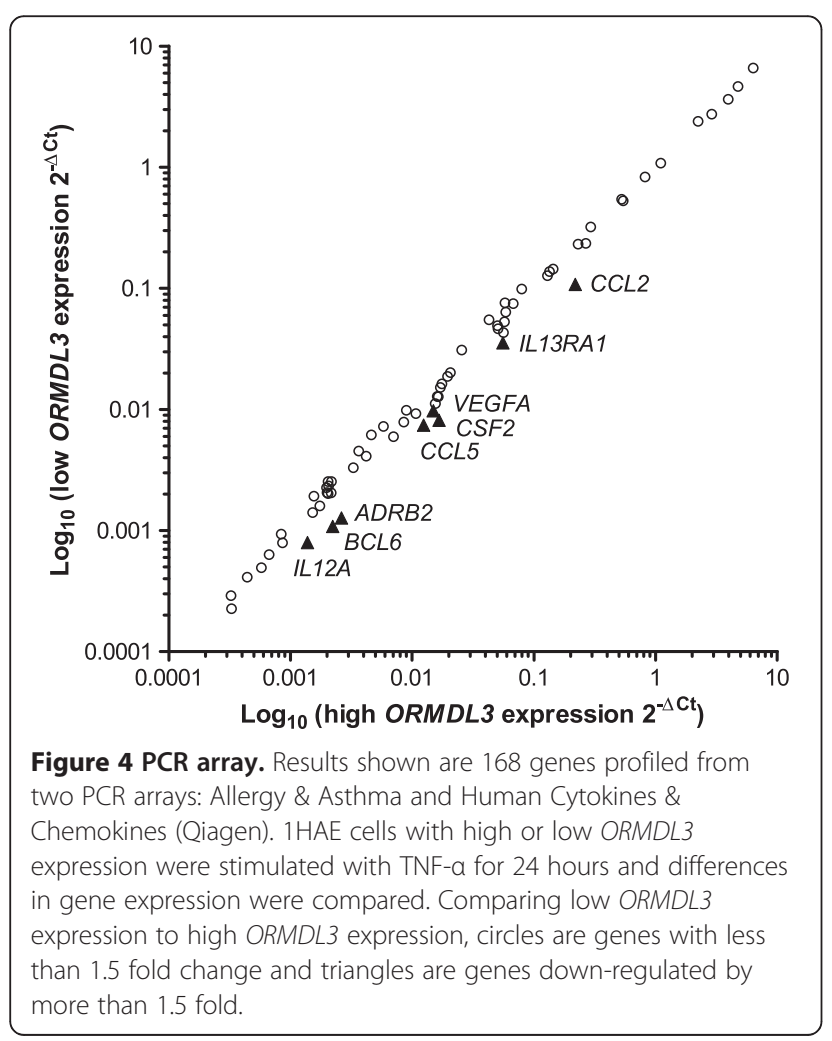

and amplified at cycle $<30$. Replication and comparison of IL23A expression between knockdown conditions yielded no significant difference. No other genes, as identified in the previous arrays, were found to be differentially regulated at this time point. These results suggest that $O R M D L 3$ variation does not have a meaningful impact on expression of a large panel of immune-related genes in airway epithelial cells.

\section{Discussion}

Asthma is a complex disease affecting many individuals in the developed world. Genome-wide association studies have recently been used to identify genetic causes for such complex diseases. One particular gene, ORMDL3, is of interest because of its association with asthma, IBD, and Type I diabetes - all of which are caused by immune-mediated pathology $[6,10,22,38,39]$. The gene $O R M D L 3$ is an ER-membrane protein and is potentially involved in $\mathrm{Ca}^{2+}$-signaling in the ER and sphingolipid synthesis $[20,21,40]$. It has also been correlated to activation of the UPR, though the mechanisms remain unclear [21]. Activation of the UPR may be biologically relevant, as ER stress, the UPR, and inflammation have all been linked [23]. However, the functional role ORMDL3 in the pathogenesis of asthma has yet to be elucidated.

Airway epithelial cells play an important role in innate immunity and in the development of asthma. Current findings in literature indicate that ORMDL3 is involved in immunity and that asthmatics have higher expression of the gene than non-asthmatics [18,21,22]. A recent study by Miller et al. also investigated the role of ORMDL3 in airway epithelial cells. They reported that in vitro overexpression of ORMDL3 activated the ATF6 pathway of the UPR and induced expression of several genes with potential importance in the pathogenesis of asthma [28]. Our investigation, in contrast, focuses on the effect of variation of ORMDL3 expression levels, at baseline, on the innate immune responsiveness of airway epithelial cells. By manipulating ORMDL3 expression in vitro to mimic differences in gene expression established between asthmatics and healthy individuals, we aimed to understand the role of ORMDL3 on the innate immune response and UPR activation status in airway epithelial cells. This method ensured control and the confidence that any effect on the innate immune response was in fact correlated with a change in ORMDL3 expression levels. If the same experiments were performed on ex vivo airway cells of patients, genetic and other differences between individuals could have affected the results.

After knockdown of ORMDL3 in vitro, cells were stimulated with cytokines (TNF- $\alpha$, IL-1 $\beta$ ) or common microbial components (LPS, flagellin). We monitored production of interleukin-6 (IL-6) and interleukin-8 (IL-8) (alias CXCL8), two pro-inflammatory cytokines produced by airway cells 
that are relevant in asthma pathogenesis. Specifically, IL-6 is elevated in individuals with asthma [41] and is also regulated by ATF6 during activation of the UPR [42]. Similarly, transfection of ORMDL3 into human airway epithelial cells triggers ATF6 activation and IL-8 secretion [28]. However, in our experimental system, variation in ORMDL3 expression levels did not affect NF-kB-induced innate immune production of IL-6 and IL- 8 in airway epithelial cells.

We next explored the effects of ORMDL3 expression on activation of the UPR. UPR signaling cascades are initiated in response to ER stress, and restoration of homeostasis is achieved by attenuating translation, restoring protein folding, or degrading misfolded proteins [24]. Although often associated with abnormal physiological conditions, the UPR plays a central beneficial role in normal physiology; as illustrated by the role of the UPR in terminal B cell differentiation which requires a massive increase in the biosynthetic capacity to synthesize antibodies in response to infection [43]. However, the ER stress response and UPR can also initiate inflammation through induction of cytokine production or activation of transcriptional regulators of inflammatory genes. Cytokines IL- 6 and IL- 8 are examples of genes that may be induced by UPR activation [23]. ER stress and the UPR have been implicated in many immune-related diseases including IBD, diabetes, chronic obstructive pulmonary disease (COPD), arthritis, and neurodegenerative inflammatory diseases [44]. It is poorly understood whether ER stress is an underlying cause of disease or if its induction is a result of chronic inflammation. Indeed, it is possible that environment factors such as infection or inhalation of smoke particles can activate the UPR, triggering the onset of lung disease in genetically predisposed individuals [45]. However, it is also possible that ER stress is exacerbated by inflammation and contributes to the perpetuation of the disease.

Cantero-Recasens et al. previously reported that ORMDL3 overexpression activated the PERK pathway, but did not affect the IRE1 pathway of the UPR [21]. In contrast, Miller et al. reported that ORMDL3 overexpression activated the ATF6 pathway, but not the PERK or IRE1 pathways [28]. In our study, we chose four markers of UPR activation: $X B P-1 u, X B P-1 s, C H O P$, and p-eIF $2 \alpha$. With activation of the UPR, we expect downregulation of $X B P-1 u$ and upregulation of XBP-1s and CHOP. However, our results demonstrate that knockdown of ORMDL3 does not activate the UPR, in either unstimulated or stimulated cells. Immunoblot analysis also showed no change in p-eIF2 $\alpha$ levels with ORMDL3 knockdown. Furthermore, downstream markers of UPR activation, IL-6 and IL- 8 cytokines, were produced at similar levels in unstimulated cells with varying ORMDL3 levels. This further supports our results that ORMDL3 does not activate the UPR. Differences in our results compared to previous work might be due to the different types of cells, conditions, or markers used. It is possible that the effects of variation in ORMDL3 expression are a cell typedependent phenomenon. While no effect on the inflammatory response was detected in airway cells, other cells types such as dendritic cells or T cells may be affected by altered ORMDL3 expression. Observations made by Lluis et al. suggest that the $17 \mathrm{q} 21$ locus may potentially play a role in T-cell development [18].

Taking a broader approach, PCR arrays looking at expression of 168 common immunity genes were performed. We reasoned that although ORMDL3 levels may not affect the production of IL-6 or IL-8 cytokines, perhaps they were impacting gene expression of other important immune genes, such as $I L-33, I L-25$ and TSLP, which have all been implicated in asthma pathogenesis [46]. Verification of differential expression of these genes at a transcript level, however, did not show any significant changes between the ORMDL3 knockdown conditions. This suggests that altering ORMDL3 expression does not have a profound effect on the expression of innate immune genes upon stimulation in the airway epithelia. However, there may be other genes that are affected that were not investigated in this study. Pfeifer et al. recently showed that IL-17C cytokine is expressed by human bronchial epithelial cells and is induced by bacterial infection [47]. It may be worthwhile in future experiments to investigate a broader range of immune-related genes. Interestingly, we did not observe changes to expression of the genes reported by Miller et al., MMP-9, CCL-20, CXCL$10, C X C L-11$, or $I L-8$. This variance may be explained by differences in experimental conditions. Our study examined outcomes in gene expression after stimulation of cells co-transfected with ORMDL3 and ORMDL3-specific siRNA, while the other study used a different experimental approach.

Although this study focused exclusively on the potential role of ORMDL3 in asthma pathogenesis, it is possible that neighboring genes such as GSDML contribute to disease susceptibility at this locus. Many groups consider ORMDL3 as an 'asthma gene'; however, it should be acknowledged that the identified SNPs associating this gene to asthma susceptibility are not located in the gene itself. Even so, these polymorphisms have been consistently correlated with increased odds of asthma risk, highlighting the importance of this locus in disease susceptibility [6-11,13,14].

Our data show that variation in ORMDL3 expression is not correlated with differential innate immune responses to stimuli or activation of the UPR in vitro in airway epithelial cells. Taken together, our results are biologically relevant because they suggest that normal human variation of ORMDL3 expression is not likely an important factor in increasing the innate immune response of airway cells we observe in asthmatics. Despite these results, this 
gene remains an important candidate for asthma susceptibility. More research is required to elucidate its role in asthma pathogenesis and its potential role as an initial trigger of inflammation. By increasing our understanding of the mechanisms responsible for allergic and atopic disease development, new treatments can then be developed. Thus, we can reduce inflammatory responses by targeting the potential triggers, rather than the symptoms, of the disease. In doing so, we will ultimately reduce the morbidity, mortality, and socio-economic burden of asthma and related allergic diseases.

\section{Additional files}

\section{Additional file 1: Genes analyzed by PCR array.}

Additional file 2: Additional figures.

\section{Competing interests}

The authors declare that they have no competing interests.

\section{Authors' contributions}

$\mathrm{KJH}$ performed the research. All authors designed the research, analyzed the data, and drafted the manuscript. Both authors read and approved the final manuscript.

\section{Acknowledgements}

$\mathrm{KJH}$ was funded by the Child \& Family Research Institute and AllerGen Networks of Centers of Excellence. SET holds the Aubrey J Tingle Professorship in Pediatric Immunology and is a clinical scholar of the Michael Smith Foundation for Health Research. This work was supported by funding from the AllerGen Networks of Centers of Excellence.

Received: 3 December 2012 Accepted: 7 January 2013

Published: 1 February 2013

\section{References}

1. Cho SH: Pharmacogenomic approaches to asthma treatment. Allergy Asthma Immunol Res 2010, 2(3):177-182.

2. Davies DE, Djukanovic R, Holgate ST: Application of functional genomics to study of inflammatory airways disease. Thorax 1999, 54(1):79-81.

3. Gu ML, Dong XQ, Zhao J: New insight into the genes susceptible to asthma. J Asthma 2010, 47(2):113-116.

4. Ober $C$, Hoffjan S: Asthma genetics 2006: the long and winding road to gene discovery. Genes Immun 2006, 7(2):p. 95-p. 100

5. Vercelli D: Discovering susceptibility genes for asthma and allergy. Nat Rev Immunol 2008, 8(3):169-182.

6. Moffatt MF, et al: Genetic variants regulating ORMDL3 expression contribute to the risk of childhood asthma. Nature 2007. 448(7152):470-473.

7. Galanter J, et al: ORMDL3 gene is associated with asthma in three ethnically diverse populations. Am J Respir Crit Care Med 2008, 177(11):1194-1200

8. Halapi $\mathrm{E}$, et al: A sequence variant on $17 q 21$ is associated with age at onset and severity of asthma. Eur J Hum Genet 2010, 18(8):902-908.

9. Hirota T, et al: Genetic polymorphism regulating ORM1-like 3 (Saccharomyces cerevisiae) expression is associated with childhood atopic asthma in a Japanese population. J Allergy Clin Immunol 2008, 121(3):769-770.

10. Moffatt MF, et al: A large-scale, consortium-based genomewide association study of asthma. N Engl J Med 2010, 363(13):1211-1221.

11. Ferreira MA, et al: Association between ORMDL3, IL1RL1 and a deletion on chromosome 17q21 with asthma risk in Australia. Eur J Hum Genet 2011, 19(4):458-464.

12. Sleiman PM, et al: ORMDL3 variants associated with asthma susceptibility in North Americans of European ancestry. J Allergy Clin Immunol 2008, 122(6):1225-1227.
13. Tavendale $\mathrm{R}$, et al: A polymorphism controlling ORMDL3 expression is associated with asthma that is poorly controlled by current medications. J Allergy Clin Immunol 2008, 121(4):860-863.

14. Wu H, et al: Genetic variation in ORM1-like 3 (ORMDL3) and gasderminlike (GSDML) and childhood asthma. Allergy 2009, 64(4):629-635.

15. Barrett JC, et al: Genome-wide association study and meta-analysis find that over 40 loci affect risk of type 1 diabetes. Nat Genet 2009, 41(6):703-707.

16. Barrett JC, et al: Genome-wide association defines more than 30 distinct susceptibility loci for Crohn's disease. Nat Genet 2008, 40(8):955-962

17. Neurath MF, Finotto $S$, Glimcher $L H$ : The role of Th1/Th2 polarization in mucosal immunity. Nat Med 2002, 8(6):567-573.

18. Lluis A, et al: Asthma-associated polymorphisms in 17q21 influence cord blood ORMDL3 and GSDMA gene expression and IL-17 secretion. J Allergy Clin Immunol 2011, 127(6):p. 1587-p. 1594. e6.

19. Jin R, et al: Mechanisms elevating ORMDL3 expression in recurrent wheeze patients: role of Ets-1, p300 and CREB. Int I Biochem Cell Biol 2012, 44(7):1174-1183.

20. Hjelmqvist $L$, et al: ORMDL proteins are a conserved new family of endoplasmic reticulum membrane proteins. Genome Biol 2002, 3(6):p. RESEARCH0027.

21. Cantero-Recasens $G$, et al: The asthma-associated ORMDL3 gene product regulates endoplasmic reticulum-mediated calcium signaling and cellular stress. Hum Mol Genet 2009, 19(1):111-121.

22. McGovern DP, et al: Genome-wide association identifies multiple ulcerative colitis susceptibility loci. Nat Genet 2010, 42(4):332-337.

23. Hotamisligil GS: Endoplasmic reticulum stress and the inflammatory basis of metabolic disease. Cell 2010, 140(6):900-917.

24. McGuckin MA, et al: ER stress and the unfolded protein response in intestinal inflammation. Am J Physiol Gastrointest Liver Physiol 2010, 298(6):G820-G832

25. Cullinan $S B$, Diehl JA: Coordination of ER and oxidative stress signaling: the PERK/Nrf2 signaling pathway. Int I Biochem Cell Biol 2006, 38(3):317-332

26. Deng J, et al: Translational repression mediates activation of nuclear factor kappa B by phosphorylated translation initiation factor 2. Mol Cell Biol 2004, 24(23):10161-10168.

27. Hu P, et al: Autocrine tumor necrosis factor alpha links endoplasmic reticulum stress to the membrane death receptor pathway through IRE1 alpha-mediated NF-kappaB activation and down-regulation of TRAF2 expression. Mol Cell Biol 2006, 26(8):3071-3084.

28. Miller $\mathrm{M}$, et al: ORMDL3 is an inducible lung epithelial gene regulating metalloproteases, chemokines, OAS, and ATF6. Proc Natl Acad Sci USA 2012, 109(41):16648-16653

29. Kim HY, DeKruyff RH, Umetsu DT: The many paths to asthma: phenotype shaped by innate and adaptive immunity. Nat Immunol 2010, 11(7):577-584.

30. Umetsu DT, et al: Asthma: an epidemic of dysregulated immunity. Nat Immunol 2002, 3(8):715-720.

31. Palmberg L, et al: Induction of IL-8 production in human alveolar macrophages and human bronchial epithelial cells in vitro by swine dust. Thorax 1998, 53(4):260-264.

32. Shanks KK, et al: Interleukin-8 production by human airway epithelial cells in response to Pseudomonas aeruginosa clinical isolates expressing type a or type b flagellins. Clin Vaccine Immuno/ 2010, 17(8):1196-1202.

33. Yang $Y$, et al: Regulation of interleukin-1 beta and interleukin-1 beta inhibitor release by human airway epithelial cells. Eur Respir J 2004, 24(3):360-366

34. Nagaraju $\mathrm{K}$, et al: A variety of cytokines and immunologically relevant surface molecules are expressed by normal human skeletal muscle cells under proinflammatory stimuli. Clin Exp Immunol 1998, 113(3):407-414

35. Pfaffl MW: A new mathematical model for relative quantification in realtime RT-PCR. Nucleic Acids Res 2001, 29(9)::45.

36. Isler JA, Skalet AH, Alwine JC: Human cytomegalovirus infection activates and regulates the unfolded protein response. J Virol 2005, 79(11):6890-6899.

37. Samali $A$, et al: Methods for monitoring endoplasmic reticulum stress and the unfolded protein response. Int I Cell Biol 2010, 2010:830307.

38. Lees CW, et al: New IBD genetics: common pathways with other diseases. Gut 2011, 60(12):1739-1753.

39. Saleh NM, et al: Genetic association analyses of atopic illness and proinflammatory cytokine genes with type 1 diabetes. Diabetes Metab Res Rev 2011, 27(8):838-843. 
40. Han S, et al: Orm1 and Orm2 are conserved endoplasmic reticulum membrane proteins regulating lipid homeostasis and protein quality control. Proc Natl Acad Sci USA 2010, 107(13):5851-5856.

41. Broide DH, et al: Cytokines in symptomatic asthma airways. J Allergy Clin Immunol 1992, 89(5):958-967.

42. Shi Y, et al: Role of GRP78/BiP degradation and ER stress in deoxynivalenol-induced interleukin-6 upregulation in the macrophage. Toxicol Sci 2009, 109(2):247-255.

43. Ma Y, Hendershot LM: The stressful road to antibody secretion. Nat Immunol 2003, 4(4):310-311.

44. Hasnain $\mathrm{SZ}$, et al: The interplay between endoplasmic reticulum stress and inflammation. Immunol Cell Biol 2012, 90(3):260-270.

45. Adair-Kirk TL, Atkinson JJ, Senior RM: Smoke particulates stress lung cells. Nat Med 2008, 14(10):1024-1025.

46. Paul WE, Zhu J: How are $\mathrm{T}(\mathrm{H}) 2$-type immune responses initiated and amplified? Nat Rev Immunol 2010, 10(4):225-235.

47. Pfeifer P, et al: IL-17C is a Mediator of Respiratory Epithelial Innate Immune Response. Am J Respir Cell Mol Biol 2012, [Epub ahead of print].

doi:10.1186/1710-1492-9-4

Cite this article as: Hsu and Turvey: Functional analysis of the impact of ORMDL3 expression on inflammation and activation of the unfolded protein response in human airway epithelial cells. Allergy, Asthma \& Clinical Immunology 2013 9:4.

\section{Submit your next manuscript to BioMed Central and take full advantage of:}

- Convenient online submission

- Thorough peer review

- No space constraints or color figure charges

- Immediate publication on acceptance

- Inclusion in PubMed, CAS, Scopus and Google Scholar

- Research which is freely available for redistribution 